\title{
Timing Acquisition with Cooperation of Two Distributed Receive Antennas over Flat-Fading Channels
}

\author{
Chaojin Qing, Jincheng Wei, Ling Xia \\ School of Electrical and Information Engineering, \\ Xihua University \\ Chengdu, Sichuan, 610039, China \\ qingchj@uestc.edu.cn
}

\begin{abstract}
In the flat Rayleigh channels of linear cell, two distributed receive antennas are employed to receive the signal transmitted from the mobile station (MS) with a single antenna. We exploit the false alarm probability at the central processor to guarantee that the false alarm probability at each distributed antenna does not exceed the pre-defined probability of false alarm. Based on the exploited probability of false alarm at the central processor, a cooperative detection threshold of each antenna is derived for threshold detection. According to the threshold detection, a maximum-likelihood (ML)-based timing acquisition method is proposed for distributed antenna systems (DAS). Without increasing the pre-defined probability of false alarm, the analysis and simulation results show that the correct acquisition probability and the missed detection probability for each distributed antenna can be improved with the proposed method wherever the MS is located.
\end{abstract}

Keywords- cooperative timing acquisition; distributed antenna systems; maximum-likelihood; threshold detection; linear cell; flat-fading channel

\section{INTRODUCTION}

Multi-antenna system can achieve higher data rates and better coverage than their single antenna counterparts without requiring additional power or bandwidth consumption [1]. However, the potential advantages of using multiple antennas cannot realize for centralized antenna systems (CAS) due to the co-located placement of antennas [1][2]. Relative to the CAS, the distributed antenna systems (DAS) presents many advantages, e.g., expanding coverage and increasing sum rates by having distributed antenna ports throughout a cell [2]-[4]. As the key technology of the next generation wireless system, the DAS has been attracting worldwide research interests [5][6].

Meanwhile, the inaccuracies of timing acquisition degrade the performance of wireless communication systems [7]-[9]. For DAS, the performance of timing acquisition is particularly important due to the different placement locations of the distributed antennas. To improve the performance of timing acquisition, some methods are proposed in [10]-[14]. However, the cooperative processing among distributed receive antennas is not exploited for timing acquisition in [10]-[12]. Although the cooperative processing is employed in [13] and [14], the transmitted signal from mobile station (MS) is assumed always exists,

\author{
Ying Liu, Chuanhui Ma, Youxi Tang \\ National Key Laboratory and Technology on \\ Communications, University of Electronic Science and \\ Technology of China, Chengdu,Sichuan,611731, China \\ \{liuying850613,tangyx \}@uestc.edu.cn
}

thus the distributed receive antennas do not detect the existence of the transmitted signal. This assumption in [13] and [14] is too ideal to be adopted for the engineering application.

To enhance the adaptability of the method in [13] and [14], a cooperative timing acquisition method, which is based on threshold detection, is proposed in this paper. For simplifying the research, two distributed receive antennas, which are placed in the flat-fading channels of linear cell [15], are employed to receive the signal transmitted from the MS with a single antenna. The false alarm probability at the central processor is exploited to guarantee that the false alarm probability at each distributed antenna does not exceed the pre-defined probability of false alarm. With the exploited probability of false alarm at the central processor, we derive a cooperative detection threshold for the two receive antennas. According to the threshold detection, a maximumlikelihood (ML)-based timing acquisition method is proposed. Through Monte Carlo simulations, we show the probability of correct acquisition and the missed detection probability for each distributed receive antenna can be improved without increasing the pre-defined probability of false alarm wherever the MS is located.

The rest of this paper is organized as follows. In the next section, we describe the system model. In Section III, we present the ML timing acquisition method. Some numerical results are given in Section IV. Finally, Section V concludes this paper.

\section{SYSTEM MODEL}

Figure 1. shows the system model, where the receive antennas $\mathrm{DRX}_{1}$ and $\mathrm{DRX}_{2}$ are assumed to receive the signal transmitted from the MS with a single antenna in the linear cell. $x(x \in[0, R]), 0$ and $R$ are respectively denoted the locations of MS, $\mathrm{DRX}_{1}$ and $\mathrm{DRX}_{2}$. The heights of MS, $\mathrm{DRX}_{1}$ and $\mathrm{DRX}_{2}$ are $h_{\mathrm{MS}}, h_{1}$ and $h_{2}$, respectively.

We denote $c(t)=\sum_{j=0}^{L-1} c_{j} g\left(t-j T_{c}\right)$ as the pulse train, where $\left\{c_{j}\right\}$ denotes the training sequence of period $L$. $g(t)$ is a unit rectangular pulse function of duration $T$ centered at $T / 2$. The transmitted baseband signal from the 
MS is $s(t)$. The baseband form of the received signal at $\mathrm{DRX}_{1}$ and $\mathrm{DRX}_{2}$, i.e., $r_{1}(t)$ and $r_{2}(t)$, can be given by

$$
\left\{\begin{array}{l}
r_{1}(t)=\sqrt{\xi_{1}} h_{1} s\left(t-\tau_{1} T\right)+n_{1}(t) \\
r_{2}(t)=\sqrt{\xi_{2}} h_{2} s\left(t-\tau_{2} T\right)+n_{2}(t)
\end{array}\right.
$$

where $h_{i}(i=1,2)$ is a zero-mean complex Gaussian random variable with unit variance. $\sqrt{\xi_{i}}(i=1,2)$ is a power gain factor that captures the effects of mean path loss and log-normal shadow fading [16]. $n_{1}(t)$ and $n_{2}(t)$ are additive white Gaussian noise with noise power $\sigma_{i}^{2} \cdot \tau_{1} T$ and $\tau_{2} T$ are respectively the time-delays from MS to $\mathrm{DRX}_{1}$ and $\mathrm{DRX}_{2} \cdot \tau_{i}(i=1,2)$ is the time-delay normalized to the period $T$.

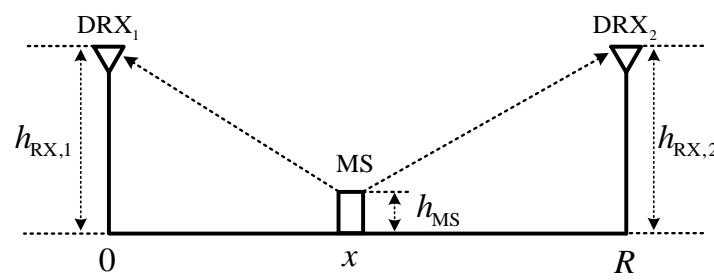

Figure 1. System model

\section{TIMING ACQUISITION}

\section{A. Detection Threshold}

At $\mathrm{DRX}_{i}$, we denote $C_{i}\left(\tau_{i}\right), i=1,2$ as the crosscorrelation between the known training sequence and the received signal. According to the traditional method of timing acquisition in [17], we have

$$
C_{i}\left(\tau_{i}\right)=\int_{0}^{T_{0}} r_{i}^{*}(t) s\left(t-\tau_{i} T\right) d t, i=1,2
$$

where $T_{0}=L T$.

Based on the researches in [13], [14] and [17], the estimation of $\tau_{i}, i=1,2$, i.e., $\hat{\tau}_{i}$ can be given by

$$
\hat{\tau}_{i}=\arg \max _{\tau_{i}}\left\{\left|C_{i}\left(\tau_{i}\right)\right|^{2} \geq T_{h, i}\right\}, i=1,2
$$

where $0<\tau_{i} \leq L-1, T_{h, i}, i=1,2$ is the detection threshold of $\mathrm{DRX}_{i}$. In (3), we consider the $\mathrm{DRX}_{i}$ can pass the threshold detection only if $\max _{\tau_{i}}\left|C_{i}\left(\tau_{i}\right)\right|^{2} \geq T_{h, i}$ is satisfied. Otherwise, the missed detection occurs at $\mathrm{DRX}_{i}$ or none of signals transmitted from MS.

Denote $P_{F A}$ as the pre-defined probability of false alarm for $\mathrm{DRX}_{1}$ and $\mathrm{DRX}_{2}$, According to the reference [18], $T_{h, i}, i=1,2$ can be expressed as

$$
T_{h, i}=-2 L \sigma_{i}^{2} \ln \left(P_{F A}\right)
$$

In (4), the detection threshold is derived without the cooperation of $\mathrm{DRX}_{1}$ and $\mathrm{DRX}_{2}$. In fact, the false alarm probability at the central processor is generally more lower than the pre-defined $P_{F A}$ if $\mathrm{DRX}_{1}$ and $\mathrm{DRX}_{2}$ all can reach the detection threshold $T_{h, i}$ according to the "data fusion rule” in [19]. Thus, the false alarm probability at the central processor can guarantee that the false alarm probability at $\mathrm{DRX}_{i}$ is not higher than the pre-defined $P_{F A}$. Then, a lower detection threshold $\tilde{T}_{h, i}$, i.e., $\tilde{T}_{h, i} \leq T_{h, i}$, can be exploited for $\mathrm{DRX}_{i}$ without increasing the pre-defined false alarm probability (i.e., $P_{F A}$ ) at $\operatorname{DRX}_{i}$. In this paper, we call $\tilde{T}_{h, i}$ as cooperative detection threshold.

To derive the cooperative detection threshold $\tilde{T}_{h, i}$, the false alarm probability at $\mathrm{DRX}_{1}$ and $\mathrm{DRX}_{2}$ are respectively assumed as $P_{F A, 1}$ and $P_{F A, 2}$. Without loss of generality, we assume $P_{F A, 1}=P_{F A, 2}$ to meet the same demands of false alarm at $\mathrm{DRX}_{1}$ and $\mathrm{DRX}_{2}$. Then the false alarm probability at the central processor, i.e., $P_{F A}^{C}$, can be obtained according to the "data fusion rule" in [19]

$$
P_{F A}^{C}=P_{F A, 1} \times P_{F A, 2}=P_{F A, 1}^{2}=P_{F A, 2}^{2} \leq P_{F A}
$$

Replacing $P_{F A}$ in (4) by the maximum of $P_{F A, 1}$ or $P_{F A, 2}$, i.e., $P_{F A, 1}=P_{F A, 2}=\sqrt{P_{F A}}$, the cooperative detection threshold $\tilde{T}_{h, i}$ can be given by

$$
\tilde{T}_{h, i}=-L \sigma_{i}^{2} \ln \left(P_{F A}\right)
$$

In this paper, we discuss the timing acquisition with cooperation according to the detection threshold given in (4) and (6).

\section{B. Timing Acquisition}

Denote $\hat{\boldsymbol{\Gamma}}_{\mathrm{ML}}=\left(\hat{\tau}_{1}, \hat{\tau}_{2}\right)^{T}$ as the maximum-likelihood (ML) estimation of $\left(\tau_{1}, \tau_{2}\right)^{T}$. On the basis of [13] and [14], we have

$$
\left\{\begin{array}{l}
\hat{\boldsymbol{\Gamma}}_{\mathrm{ML}}=\arg \max _{\left\{\tau_{1}, \tau_{2}\right\}}\left\{\left|C_{1}\left(\tau_{1}\right)\right|^{2}+\left|C_{2}\left(\tau_{2}\right)\right|^{2}\right\} \\
\text { s.t. }\left|C_{1}\left(\hat{\tau}_{1}\right)\right|^{2} \geq \tilde{T}_{h, 1},\left|C_{2}\left(\hat{\tau}_{2}\right)\right|^{2} \geq \tilde{T}_{h, 2}
\end{array}\right.
$$

where $\tilde{T}_{h, i}$ is given in (6).

When $\quad\left|C_{1}\left(\hat{\tau}_{1}\right)\right|^{2} \geq \tilde{T}_{h, 1} \quad$ and $\quad\left|C_{2}\left(\hat{\tau}_{2}\right)\right|^{2} \geq \tilde{T}_{h, 2} \quad$ cannot simultaneously be satisfied, the $\mathrm{DRX}_{i}$, which can reach the detection threshold $\tilde{T}_{h, 1}$, must be verified to ensure the false alarm probability at $\mathrm{DRX}_{i}$ is not higher than the pre-defined $P_{F A}$. Assuming $\mathrm{DRX}_{j}, j=1$ or 2 can only reach the detection threshold $\tilde{T}_{h, j}$, the estimation $\hat{\tau}_{j}$ can be given by 


$$
\left\{\begin{array}{l}
\hat{\tau}_{j}=\arg \max _{\tau_{j}}\left\{\left|C_{j}\left(\tau_{j}\right)\right|^{2}\right\}, j=1 \text { or } 2 \\
\text { s.t. }\left|C_{j}\left(\hat{\tau}_{j}\right)\right|^{2} \geq T_{h, j}
\end{array}\right.
$$

where $T_{h, j}$ can be obtained from (4)

\section{NUMERICAL AND SIMULATION RESUlTS}

To confirm the effectiveness of the proposed method, computer simulation results are presented in this section. Some basic simulation approaches and parameters are as follows: $R=500 \mathrm{~m}, h_{1}=h_{2}=6 \mathrm{~m}, h_{\mathrm{MS}}=1 \mathrm{~m}$, the probability of pre-defined false alarm $P_{F A}=10^{-6}$, the training sequence is Zadoff-Chu sequence [20], which is also adopted by the 3rd Generation Partnership Project (3GPP) Long Term Evolution (LTE) standard. $L=1024, T=1 / 1.5 \mu \mathrm{s}, \sigma_{1}^{2} \approx \sigma_{2}^{2}$, the detection threshold is set according to (4) and (6). From reference [21], we consider the path loss and shadowing model, i.e.,

$$
\left(\frac{P_{r, i}}{P_{t}}\right)_{d B}=10 \lg K-10 \gamma \lg \left(\frac{d_{i}}{d_{0}}\right)-\varphi_{d B}, i=1,2
$$

where $P_{t}$ is the transmitted power of MS, $P_{r, i}$ is the received power of $\mathrm{DRX}_{i}, K=\left(\lambda / 4 \pi d_{0}\right)^{2}$ (where $\lambda$ is wavelength of carrier wave and $d_{0}$ is a reference distance for the antenna far-field), $\gamma$ is the path loss exponent, $\varphi_{d B}$ is a Gauss distributed random variable with mean zero and variance $\sigma_{\varphi_{d B}}^{2}, d_{i}$ is the distance from MS to $\mathrm{DRX}_{i}$, which can be expressed as

$$
\left\{\begin{array}{l}
d_{1}=\sqrt{\left(h_{\mathrm{RX}, 1}-h_{\mathrm{MS}}\right)^{2}+x^{2}} \\
d_{2}=\sqrt{\left(h_{\mathrm{RX}, 2}-h_{\mathrm{MS}}\right)^{2}+(R-x)^{2}}
\end{array}\right.
$$

From [21], we set $\gamma=3, d_{0}=1 \mathrm{~m}, \sigma_{\varphi_{\mathrm{dB}}}=3.7$, $\lambda=0.15 \mathrm{~m}$ (i.e., the carrier frequency is $2 \mathrm{GHz}$ ). The noise power at $\mathrm{DRX}_{i}$ is set as $-100 \mathrm{dBm}$ and the transmitted power of MS is $P_{t}=-20 \mathrm{dBm}$. Assume the transmitted power of MS keeps unchanged wherever the MS is located in the linear cell (i.e., $x \in[0, R]$ ).

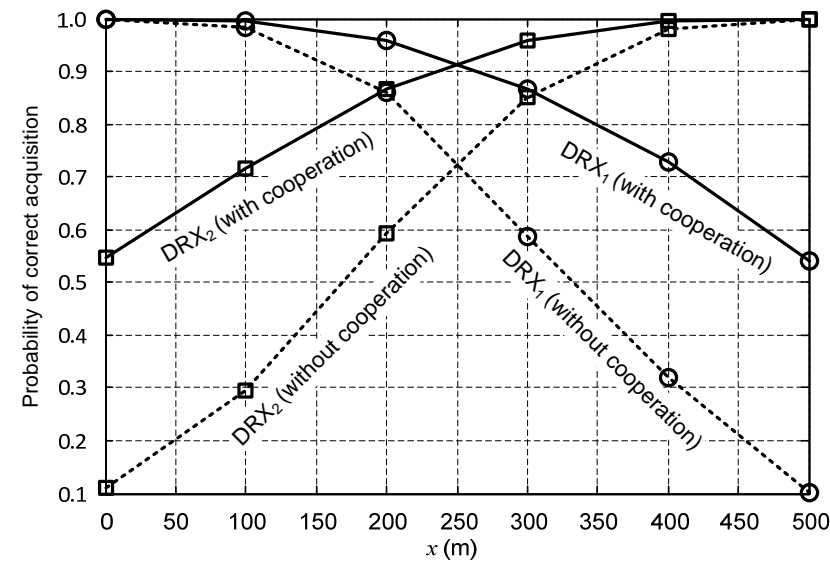

Figure 2. The probability of correct acquisition versus MS location $X$

$$
\left(P_{t}=-20 \mathrm{dBm}\right)
$$

Figure 2. gives the correct acquisition probability versus MS location $x$ when $P_{t}=-20 \mathrm{dBm}$. From Figure 2. the probability of correct acquisition for each antenna is improved with their cooperation. For the case that the location of MS is $x=250 \mathrm{~m}$, the correct acquisition probability of $\mathrm{DRX}_{1}$ and $\mathrm{DRX}_{2}$ is only $72 \%$ without cooperation, while it increase to $91 \%$ with the cooperation of $\mathrm{DRX}_{1}$ and $\mathrm{DRX}_{2}$.

In Figure 2. , when $x=0 \mathrm{~m}$ (or $x=500 \mathrm{~m}$ ), i.e., the MS is close to $\mathrm{DRX}_{1}$ (or $\mathrm{DRX}_{2}$ ) and far away from $\mathrm{DRX}_{2}$ (or $\mathrm{DRX}_{1}$ ), the probability of correct acquisition for $\mathrm{DRX}_{1}$ (or $\mathrm{DRX}_{2}$ ) keeps it nearly $100 \%$. Thus, the proposed method has its robustness.

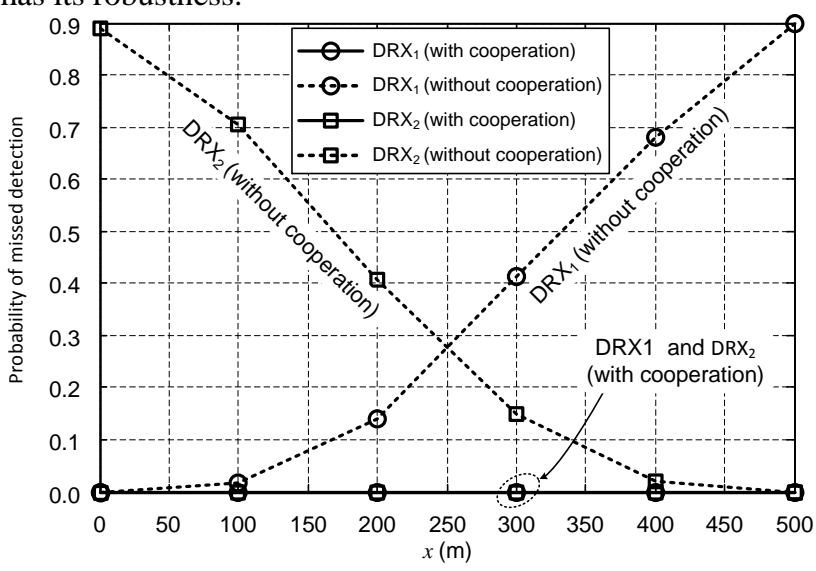

Figure 3. The probability of missed detection versus MS location $X$

$$
\left(P_{t}=-20 \mathrm{dBm}\right)
$$

Figure 3. plots the missed detection probability versus MS location $x$. From Figure 3., the missed detection probability is decrease dramatically with the cooperation of $\mathrm{DRX}_{1}$ and $\mathrm{DRX}_{2}$. With the cooperation between the two receive antennas, i.e., $\mathrm{DRX}_{1}$ and $\mathrm{DRX}_{2}$, the missed detection probability of $\mathrm{DRX}_{1}$ and $\mathrm{DRX}_{2}$ all can decrease 
to far below $10 \%$, while the missed detection probability of $\mathrm{DRX}_{2}$ (or $\mathrm{DRX}_{1}$ ) reaches $90 \%$ without the cooperation of $\mathrm{DRX}_{1}\left(\right.$ or $\mathrm{DRX}_{2}$ ) when $x=0 \mathrm{~m}$ (or $x=500 \mathrm{~m}$ ). Relative to the cases without cooperation, the simulation results in Figure 3. show that the missed detection probability is almost always improved.

\section{CONCLUSION}

We have investigated a timing acquisition method for distributed antenna systems to enhance the adaptability of the existing methods of cooperative timing acquisition. In the flat Rayleigh channels of linear cell, the false alarm probability at the central processor is exploited to guarantee the false alarm probability at each distributed antenna does not exceed the pre-defined probability of false alarm. Based on the exploited probability of false alarm at the central processor, a cooperative detection threshold for each antenna is derived for threshold detection. According to the threshold detection, a maximum-likelihood (ML)-based timing acquisition method is proposed. Without increasing the predefined probability of false alarm, the Monte Carlo simulations results show that the correct acquisition probability and the missed detection probability for each distributed antenna can be improved with the proposed method wherever the MS is located.

\section{ACKNOWLEDGMENT}

This work is supported in part by the National Natural Science Foundation under Grant number 60832007, 60901018, 60902027, U1035002/L05, 61001087, 863 Project under Grant number 2009AA01Z236, the National major projects under Grant number 2010ZX03003-002-01, 2011ZX03001- 006-01, the Fundamental Research Funds for the Central Universities under Grant number ZYGX2009J008, ZYGX2009J010, the key projects of Xihua University under Grant number Z1120941, the key projects of Education Department of Sichuan Province under Grant number 12ZA161, the Chunhui plan of Ministry of education under Grant number Z2011091, and the Science Foundation for Post Doctorate Research under Grant number 2012M511920 of China.

\section{REFERENCES}

[1] R. H. Gohary, H. Yanikomeroglu, S. Al-Ahmadi, and G. Boudreau. Coordinated port selection and beam steering optimization in a multi-cell distributed antenna system using semidefinite relaxation. IEEE Trans. Wireless Commun., 2012, 11(5): 1861-1871.

[2] H. Kim, S.-R. Lee, K.-J. Lee, and I. Lee. Transmission schemes based on sum rate analysis in distributed antenna systems. IEEE Trans.
Wireless Commun., 2012, 11(3): 1201-1209.

[3] J. Park, E. Song, W. Sung. Capacity analysis for distributed antenna systems using cooperative transmission schemes in fading channels. IEEE Trans. Wireless Commun., 2009, 8(2): 586-592.

[4] M. Dohler, H. Aghvami. Information outage probability of distributed STBCs over Nakagami fading channels. IEEE Commun. Lett., 2004, 8(7): 437-439.

[5] J. Z. Wang, H. L. Zhu; N. J. Gomes. Distributed antenna systems for mobile communications in high speed trains. IEEE J. Sel. Areas Commun. 2012, 30(4): 675-683.

[6] E. S. Park, S. R. Lee, I. Lee. Antenna placement optimization for distributed antenna systems. IEEE Trans. Wireless Commun. 2012, 11(7): 2468-2477.

[7] P. K. Vitthaladevuni and M.-S. Alouini. Effects of imperfect phase and timing synchronization on the bit-error rate performance of PSK modulations. IEEE Trans. Commun. 2005, 53(7): 1096-1099.

[8] Y. Mostofi and D. C. Cox. Mathematical analysis of the impact of timing synchronization errors on the performance of an OFDM system. IEEE Trans. Commun. 2006, 54(2): 226-230.

[9] H. O. R. Muhammad, S. P. Majumder. Effect of timing synchronization error on the bit error rate performance of an OFDM system. in Proc. IEEE Information and Communication Technology (ICICT’2007), Dhaka, 2007, 227-230.

10] C. Feng, J. Zhang, Y. Zhang, M. Xia. A novel timing synchronization method for MIMO OFDM systems. in Proc. IEEE Vehicular Technology Conference (VTC'2008-Spring), Singapore, 2008, 1443-1447.

11] G. Liu, J. Ge, Y. Guo. Time and frequency offset estimation for distributed multiple-input multiple-output orthogonal frequency division multiplexing systems. IET Commun. 2010, 4(6): 708-715.

12] Y. Y. Zhang, J. H. Zhang, F. F. Sun, et al. A novel timing synchronization method for distributed MIMO-OFDM systems in multipath Rayleigh fading channels. in Proc. IEEE Vehicular Technology Conference (VTC'2008-Spring), Singapore, 2008, 1443-1447.

13] Chaojin Qing, Shihai Shao, Youxi Tang. Timing acquisition for distributed antenna systems by exploiting the advantages of cooperation over flat-fading channels. Chinese Journal of Electronics, 2011, 20(2):337342.

[14] Chaojin Qing, Shihai Shao, Youxi Tang. Timing acquisition for distributed antenna systems with the cooperation of two remote antennas. Chinese Journal of Electronics, 2011, 20(3):527-532.

15] Y. Shen, Y. X. Tang, T. Kong, et al. Optimal antenna location for STBC-OFDM downlink with distributed transmit antennas in linear cells. IEEE Commun. Lett. 2007, 11(5): 387-389.

[16] T. S. Rappaport. Wireless communications: principles and practice. 2nd ed. New York: Addison Wesley/Pearson, 2004: 105-248.

[17] R. Pickholiz, D. Schilling, and L. Milstein. Theory of spreadspectrum communications-A tutorial. lEEE Trans. Commun. 1982, 30(5): 855-884.

[18] A. B. Awoseyila, C. Kasparis and B.G. Evans. Robust time-domain timing and frequency synchronization for OFDM systems. IEEE Trans. Consumer Electron. 2009, 55(2): 391-399.

[19] M.Barkat, "Signal detection and estimation," 2nd edition, Artech House, Inc. 2005.

20] D. C. Chu. Polyphase codes with good periodic correlation properties. IEEE Trans. Info. Theory. 1972, 18(4): 531-532.

[21] A. Goldsmith, Wireless Communications. Cambridge, U.K.: Cambridge Univ. Press, 2004. 